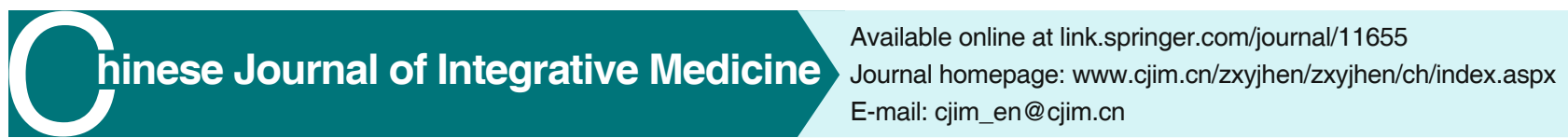

Evidence-Based Integrative Medicine

\title{
Is Chinese Medicine Injection Applicable for Treating Acute Lung Injury and Acute Respiratory Distress Syndrome? A Systematic Review and Meta-analysis of Randomized Controlled Trials*
}

\author{
CHEN Yuan-bin ${ }^{1,2,3}$, LIU Qiao ${ }^{4}$, XIE Han H,3 $^{2,3}$ YIN Shuo-miao', WU Lei ${ }^{2,3}$, \\ YU Xu-hua ${ }^{2,3}$, FAN Long ${ }^{2,3}$, and LIN Lin ${ }^{2,3,5}$
}

\begin{abstract}
Objective: To assess the efficacy and safety of Chinese medicine injection (CMI) for treating acute lung injury/acute respiratory distress syndrome (ALI/ARDS). Methods: Randomized controlled trials (RCTs) were identified by searching 3 English databases and 4 Chinese databases from their inceptions until February 2019. The Cochrane Handbook was used to evaluate risk of bias in the included studies. Data analysis was conducted using RevMan 5.3.3 software. Results: A total of 19 eligible RCTs involving 1,334 participants was included in this systematic review and meta-analysis. The main meta-analysis showed that CMI combined with conventional therapy (CT) was more effective than CT alone in reducing the acute physiology and chronic health evaluation (APACHE) II score [mean difference (MD): -1.74 points, $95 \%$ confidence interval $(\mathrm{Cl}):-2.77$ to $-0.71, I^{2}=0$ ] and increasing the total effective rate [relative risk (RR): $1.35,95 \% \mathrm{Cl}: 1.17$ to $1.56, I^{2}=37 \%$ ]. Compared with CT, CMI combined with CT showed improvements in the arterial partial pressure of oxygen $\left(\mathrm{PaO}_{2}, \mathrm{MD}: 9.25 \mathrm{~mm} \mathrm{Hg}, 95 \%\right.$ Cl: 0.87 to $\left.17.63, I^{2}=98 \%\right)$ and oxygenation index [arterial partial pressure of oxygen $\left(\mathrm{PaO}_{2}\right)$ /fraction of inspired oxygen $\left(\mathrm{FiO}_{2}\right)$, MD: $50.75 \mathrm{~mm} \mathrm{Hg}, 95 \% \mathrm{Cl}: 35.18$ to $\left.66.31, \mathrm{l}^{2}=94 \%\right]$. CMI plus $\mathrm{CT}$ was superior to $\mathrm{CT}$ in reducing the systemic inflammatory response syndrome (SIRS) score (MD: -0.84 points, $95 \% \mathrm{Cl}:-1.26$ to $-0.42, I^{2}=65 \%$ ), length of hospital stay (MD: -4.22 days, $95 \% \mathrm{Cl}:-6.49$ to $-1.95, l^{2}=92 \%$ ), and duration of mechanical ventilation (MD: -2.94 days, $95 \% \mathrm{Cl}:-4.68$ to $-1.21, l^{2}=89 \%$ ). Only 1 study reported adverse events. Conclusions: CMI as an adjuvant therapy showed great potential benefits for the treatment of ALI/ARDS. However, we could not make a definite conclusion due to low quality of included studies and uncertain security. Future studies should focus on improving research design, especially in blindness and placebo. The reporting of adverse events was also needed.
\end{abstract}

KEYWORDS Chinese medicine injection, acute lung injury, acute respiratory distress syndrome, systematic review

In 1994, the American European Consensus Conference (AECC) defined acute respiratory distress syndrome (ARDS) as acute inflammatory syndrome manifesting as diffuse pulmonary oedema and respiratory failure that cannot be explained by, but may co-exist with, left-sided heart failure. ${ }^{(1)}$ ARDS is a severe form of acute lung injury (ALI). All patients with ARDS have ALI, but not all patients with ALI have ARDS. The Berlin criteria removed the term ALI from the ARDS definition in 2012. ${ }^{(2)}$ According to the degree of hypoxaemia, ARDS is divided into 3 stages: mild, moderate and severe. The clinical manifestations of ALI/ARDS are respiratory distress, refractory hypoxemia and other symptoms. A cross-sectional study has revealed that the incidence of ALI is
CThe Chinese Journal of Integrated Traditional and Western Medicine Press and Springer-Verlag GmbH Germany, part of Springer Nature 2019

*Supported by the National Natural Science Foundation of China (Nos. 81503511 and 81503676) and Guangdong Science and Technology Project (Nos. 2017A020213007 and 2017B030314176)

1. PhD Candidate, the Second Clinical College of Guangzhou University of Chinese Medicine, Guangzhou (510403), China; 2. Department of Respiratory Medicine, the Second Affiliated Hospital of Guangzhou University of Chinese Medicine, Guangzhou (510120), China; 3. Department of Respiratory Medicine, Guangdong Provincial Hospital of Chinese Medicine, Guangzhou (510120), China; 4. Department of Internal Medicine, Heyuan Hospital of Traditional Chinese Medicine, Heyuan (517000), Guangdong Province, China; 5. ZHOU Zhong-ying Famous Doctors' Workshop of Guangdong Provincial Hospital of Chinese Medicine, Guangzhou (510120), China

Correspondence to: Prof. LIN Lin, E-mail: drlinlin620@163.com DOI: https://doi.org/10.1007/s11655-019-3078-7 
$789 / 1,000,000 .^{(3)}$ ARDS affects about 3 million people a year in the world. ${ }^{(4)}$ With the deepening understanding of ALI/ARDS and the application of lung protective ventilation strategies, the mortality rate for the disease is downward trend, but the risk of death still remains between $29 \%-42 \% .{ }^{(5)}$ Considering their complex etiology and pathogenesis, numerous pathogenic links and high mortality, ALI/ARDS seriously affects the quality of life of patients, and even threatens life. Thus, it has become a controversial topic in the field of clinical critical illness research. ${ }^{(6)}$

At present, no specific treatments for ALI/ARDS have been reported, and the general practice is to take comprehensive treatment measures, including treating the primary disease, infection control, breathing and circulation support, prevention and treatment of complications and multiple organ dysfunction syndrome, according to their pathophysiological changes and clinical manifestations. The supportive treatment of mechanical ventilation is the basis for the treatment of ARDS. ${ }^{(7)}$ In Western medicine, the drug therapies are usually based on an anti-inflammatory strategy involving antibiotics and glucocorticoids. ${ }^{(8)}$ Despite their effect in relieving acute inflammation, the anti-inflammatory strategy may weaken the immune system, increase the chances of infection, delay recovery from injury, turn acute inflammation into chronic, or increase other risks. ${ }^{(9)}$ Furthermore, long-term and large-sample control studies have shown that drug therapies do not reduce ALI/ARDS mortality. ${ }^{(10)}$

Chinese medicine (CM) has a potential advantage in the prevention and treatment of ALI/ARDS, which has become increasingly important. The research and development of Chinese medicine injection (CMI) is a major innovation in the modernization of $\mathrm{CM}$. $\mathrm{CMI}$ is effective in the treatment of intractable and acute diseases, and has been widely used for ALI/ARDS in China. ${ }^{(11)}$ For example, Xuebijing Injection (血必净注 射液) can effectively control inflammatory reactions in patients with ALI/ARDS. ${ }^{(12)}$ Tanreqing Injection (痰 热清注射液) can inhibit the release of inflammatory mediators, alleviate the exudation of alveolar inflammation, promote the synthesis of pulmonary surfactant and reduce the scope of alveolar exudation, thereby protecting the lungs from ALI. ${ }^{(13)}$ However, no systematic review has evaluated the efficacy and safety of CMI in the treatment of ALI/ARDS. Therefore, we evaluated the evidence of systematic reviews in order to recommend whether adding $\mathrm{CMI}$ is beneficial and safe for patients with ALI/ARDS.

\section{METHODS}

\section{Search Strategy}

Searches were conducted in 3 English databases (PubMed, EMBASE, and Cochrane Central Register of Controlled Trials) and 4 Chinese databases including Wanfang, Chinese BioMedical (CBM), China Knowledge Resource Integrated Database (CNKI), and VIP database from their inceptions until February 2019. The following keywords relevant to 3 groups of search terms were used to identify relevant studies: disease (ALI/ARDS and their synonyms); intervention (CMI and its synonyms) and study type [randomized controlled trials (RCTs)]. In order to identify as many trials as possible, meta-analysis, clinical trial registrations and dissertations were also included in the search.

\section{Inclusion Criteria}

Studies that met the following criteria were eligible: (1) the RCTs were qualified; (2) participants had been diagnosed with ALI/ARDS according to the AECC definition ${ }^{(1)}$ or Berlin definition; ${ }^{(2)}$ (3) patients in the intervention group had received $\mathrm{CMI}$ in combination with conventional therapy (CT), and the control group received the same $\mathrm{CT}$; (4) the components of $\mathrm{CMI}$ could be either a single Chinese herb or multiple Chinese herbs. CT was primarily respiratory support and pharmacotherapy. Oxygen therapy and mechanical ventilation are routine measures of respiratory support therapy. Pharmacotherapy is routinely used in treatments such as glucocorticoid or fluid management; (5) the outcome measures included arterial partial pressure of oxygen $\left(\mathrm{PaO}_{2}\right)$, oxygenation index $\left[\mathrm{PaO}_{2} /\right.$ fraction of inspired oxygen $\left.\left(\mathrm{FiO}_{2}\right)\right]$, systemic inflammatory response syndrome (SIRS) score, acute physiology and chronic health evaluation (APACHE) II score, duration of mechanical ventilation, length of hospital stay and total effective rate.

\section{Exclusion Criteria}

Studies were excluded if they met any of the following criteria: (1) CMI had been combined with other CM interventions such as oral Chinese herbal medicine, acupuncture or herbal enema; (2) CT not routinely recommended for ALI/ARDS were used; or (3) studies reported no related outcomes.

\section{Data Extraction}

Two researchers (Chen YB and Liu Q) screened 
the studies and extracted data independently. Controversies were mediated by a third researcher (Lin L) when necessary. EpiData 3.1 (Odense, Denmark) was used for double entry and data extraction. The extracted information was exported as a Microsoft Excel spread sheet from EpiData, and included basic characteristics and outcome data. The following items were essential: (1) basic details (e.g. title, authors, publication date); (2) participants (e.g. sample size, dropout rate, age, sex, diagnostic criteria); (3) research design (e.g. methodological evaluation, experimental and control interventions, treatment duration, outcome measures); (4) research results; and (5) adverse events.

\section{Risk of Bias Assessment}

The risk of bias was assessed independently by 2 reviewers (Chen YB and Xie $\mathrm{H}$ ) according to the Cochrane Collaboration's tool for appraising the risk of bias. ${ }^{(14)}$ The risk of bias in 7 domains was evaluated, including random sequence generation, allocation concealment, blinding of participants and personnel, blinding of outcome assessment, incomplete outcome data, selective outcome reporting, and other bias. Blindness was evaluated based on participantreported outcomes (PROs) and clinician-reported outcomes (CROs). Other bias included funding source, baseline balance, and conflicts of interest. Each risk of bias domain could be classified as low risk, unclear risk, or high risk. Attempts were made to contact corresponding authors via phone, mail, or e-mail when relevant information was missing or incomplete. Another researcher (Wu L) validated the final risk of bias if any discrepancies were found.

\section{Data Analysis}

Review manager (RevMan 5.3.3, Cochrane Collaboration) was used for statistical analysis. Continuous data were presented as mean difference (MD), with 95\% confidence intervals (Cl) between two groups, while discontinuous data were calculated as relative risk $(\mathrm{RR})$ with $95 \% \mathrm{Cl}$. Heterogeneity was assessed using $P$-values and $I^{2}$ index. An $I^{2}$ index less than or equal to $50 \%\left(I^{2} \leqslant 50 \%\right)$ was considered an indicator of low heterogeneity. If studies had a high heterogeneity, subgroup analysis and sensitivity analysis were used to explain the results.

\section{Publication Bias}

Funnel plot analysis was conducted to evaluate publication bias when there had been more than 10 studies merged in a pool. The Egger's test was conducted when funnel plot appeared visual anisomerous.

\section{RESULTS}

\section{Literature Search}

The initial retrieval identified 6,693 studies from literature databases. After removing duplicates, screening abstracts and reading full texts, 19 eligible RCTs involving 1,334 participants were included in the systematic review and meta-analysis. ${ }^{(15-33)}$ The screening process for the literature search is depicted in Appendix 1.

\section{Study Characteristics}

The 19 included studies were all single-center RCTs conducted in China between 2010 and 2018, involving a total of 1,334 participants. The sample size varied from 30 to 136 . Disease history was reported in $2^{(12,22)}$ of the trials, and the duration of treatment for both experimental and control groups ranged from 6 to 15 days. All participants had received CTs, including respiratory supports, circulation supports, antibiotics, and treatment of original diseases. Besides CTs, the treatment groups had also been treated with CMI [e.g. Tanreqing Injection, Shengmai Injection (生脉注射 液), Shenfu Injection (参附注射液), Danshen Injection (丹参注射液), Reduning Injection (热毒宁注射液), or Xuebijing Injection]. Eight studies ${ }^{(15-17,19,24,28,30,31)}$ reported the outcome of $\mathrm{PaO}_{2}, 13^{(15-17,19-21,25-28,30-32)}$ evaluated $\mathrm{PaO}_{2} / \mathrm{FiO}_{2}, 6$ appraised length of hospital stay $^{(20,23,25,26,29,32)}$ and total effective rate, ${ }^{(15,18,21,24,28,30)}$ 4 reported SIRS score ${ }^{(15-17,20)}$ and APACHE II score, ${ }^{(20,22,27,33)}$ and $7^{(20-23,25,26,32)}$ reported duration of mechanical ventilation. Characteristics of all included studies are summarized in Appendixes 2 and 3.

\section{Risk of Bias}

Eight studies $^{(15-17,19,22,26,27,30)}$ (42\%) explicitly reported adequate methods for random sequence generation, and were judged to be at low risk of selection bias. All studies that did not describe sequence allocation were judged to be of unclear risk. Blinding of participants and personnel were not performed in all studies, and they were judged as having high risk of bias. Four studies ${ }^{(19,27,29,31)}(21 \%)$ reported the outcome assessment of CROs, which were judged to be low risk, although blindness was not mentioned. Seven studies ${ }^{(15,18,20,21,24,28,30)}$ (37\%) declared that the blinding of PROs had been at high risk of detection bias. Incomplete outcome data was evaluated to be of low risk of attrition bias in all studies, because there were no missing data or the numbers and reasons 
for withdrawal in the two groups were similar. All studies were judged as having low risk of bias for selective outcome reporting. Six studies ${ }^{(17,19,21,26,31,32)}(32 \%)$ showed balance baselines and had been supported by research funding, they were judged to be of low risk of other bias. The risk of bias is summarized in Appendix 4.

\section{Publication Bias}

A $\mathrm{PaO}_{2} / \mathrm{FiO}_{2}$ funnel plot was created based on 13 studies. ${ }^{(15-17,19-21,25-28,30-32)}$ Other outcome measures were not generated because there were an inadequate number of studies. Visually, the $\mathrm{PaO}_{2} /$ $\mathrm{FiO}_{2}$ funnel plot appeared anisomerous. Therefore, an Egger's test was conducted, and no publication bias was found ( $t=1.96,95 \% \mathrm{Cl}$ : -1.07 to $18.73, P=0.07$ ). These results are shown in Appendix 5.

\section{Outcome Measures}

$\mathrm{PaO}_{2}$

In 8 studies ${ }^{(15-17,19,24,28,30,31)}$ in this review, $\mathrm{PaO}_{2}$ was evaluated by clinician, and the results of the forest plot showed a high heterogeneity (MD: $9.25 \mathrm{~mm} \mathrm{Hg}, 95 \%$ Cl: 0.87 to $17.63, I^{2}=98 \% ; 650$ participants). Subgroup analysis was conducted based on the therapeutic methods of CM. Subgroup 1 compared patients receiving $\mathrm{CMI}$ for clearing heat and detoxifying plus CT with CT in 5 studies, ${ }^{(15-17,19,30)}$ but heterogeneity was evident (MD: $14.48 \mathrm{~mm} \mathrm{Hg}, 95 \% \mathrm{Cl}: 7.49$ to 21.47 , $\mathrm{I}^{2}=95 \%$ ). Subgroup 2 compared patients receiving CMI for activating blood and resolving stasis plus CT with CT in 2 studies. ${ }^{(24,31)} \mathrm{PaO}_{2}$ increased $12.11 \mathrm{~mm} \mathrm{Hg}(95 \%$ Cl: 10.49 to $13.73, I^{2}=0,141$ participants). Subgroup 3 contained only 1 study $^{(28)}$ using $\mathrm{CMI}$ of tonifying qi and enriching yin, the result showed CMI plus CT was not superior to CT (MD: $-22.07 \mathrm{~mm} \mathrm{Hg}, 95 \% \mathrm{Cl}:-25.61$ to -18.53). The overall result showed that improvement in the $\mathrm{PaO}_{2}$ in CMI plus CT group was better than the CT alone group. These results are shown in Figure 1.

$\mathrm{PaO}_{2} / \mathrm{FiO}_{2}$

$\mathrm{PaO}_{2} / \mathrm{FiO}_{2}$ was assessed by clinician in 13 studies, ${ }^{(15-17,19-21,25-28,30-32)}$ and the forest plot showed a high heterogeneity (MD: $50.75 \mathrm{~mm} \mathrm{Hg}, 95 \% \mathrm{Cl}: 35.18$ to $66.31, I^{2}=94 \%$; 915 participants). Subgroup analysis was performed in accordance with the therapeutic methods of $\mathrm{CM}$. In the subgroup of $\mathrm{CMI}$ using the method of activating

$$
\text { Favours }[\mathrm{CMI}+\mathrm{CT}] \quad \text { Favours }[\mathrm{CT}] \quad \text { Mean Difference Mean Difference }
$$

Study or subgroup Mean SD Total Mean SD Total Weight IV, Random, $95 \% \mathrm{Cl} \quad$ IV, Random, $95 \% \mathrm{Cl}$

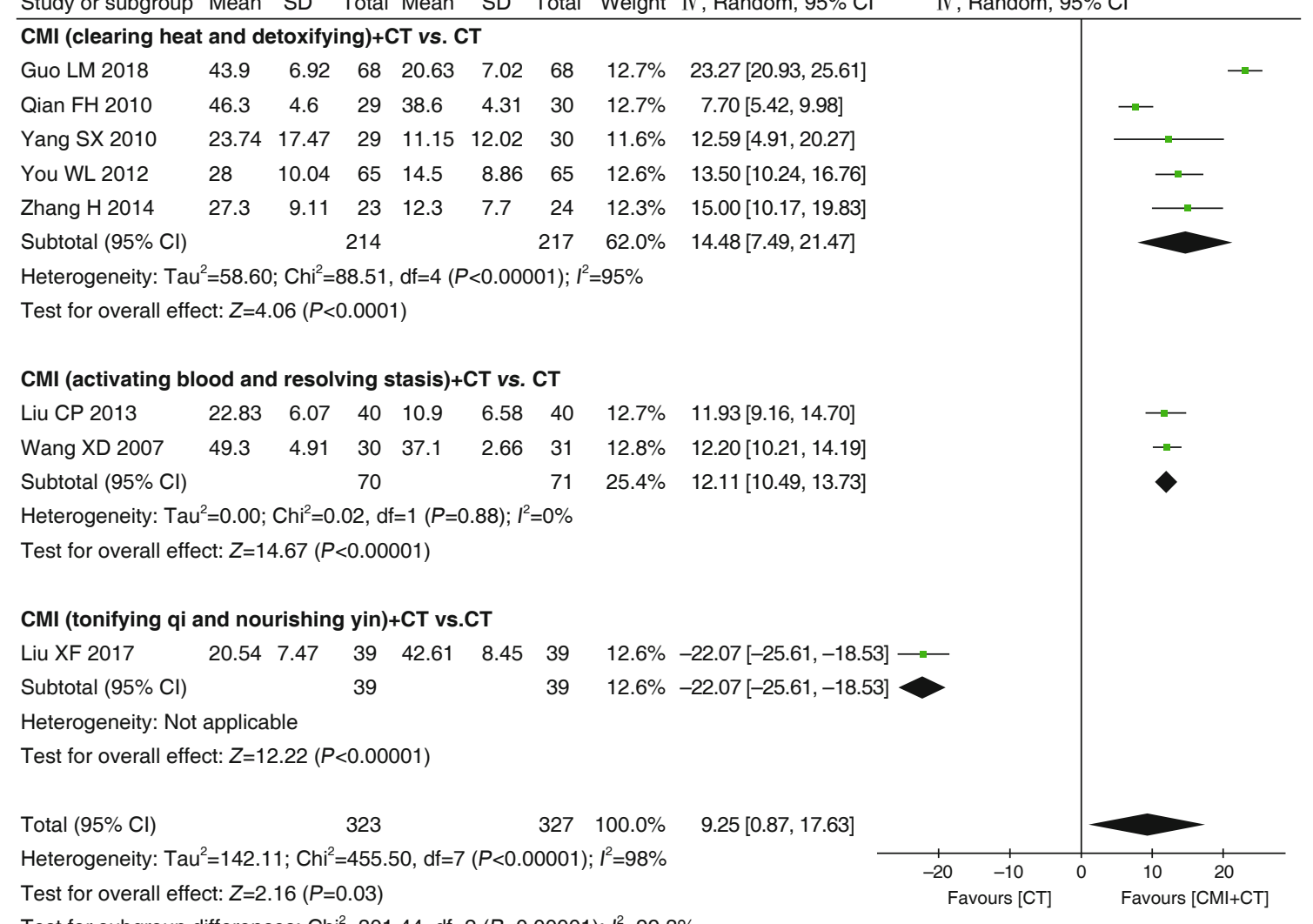

Test for subgroup differences: $\mathrm{Chi}^{2}=301.44$, df=2 $(P<0.00001) ; I^{2}=99.3 \%$

Figure 1. Comparison of $\mathrm{CMI}$ plus $\mathrm{CT}$ versus $\mathrm{CT}$ for ALI/ARDS: Change in $\mathrm{PaO}_{2}$ 
blood and resolving stasis, 2 studies $^{(31,32)}$ showed that $\mathrm{CMI}$ plus CT was better than CT alone in improving $\mathrm{PaO}_{2} / \mathrm{FiO}_{2}$, and the heterogeneity is low (95\% Cl: 16.55 to 52.92 , $I^{2}=16 \%, 110$ participants). Another subgroup of CMI using the method of tonifying qi and enriching yin also showed equally positive result ( $95 \% \mathrm{Cl}: 9.18$ to 42.57 , $I^{2}=30 \%, 125$ participants in 2 studies). ${ }^{(27,28)}$ The results of the forest plot in the other subgroups showed a high heterogeneity, possibly due to participant age or different causes of onset (i.e. infection and trauma). These results are shown in Figure 2.

\section{SIRS Score}

SIRS score was evaluated in 4 studies, ${ }^{(15-17,20)}$ and results of the forest plot showed moderate heterogeneity
(MD: -0.84 points, $95 \% \mathrm{Cl}:-1.26$ to $-0.42, I^{2}=65 \%$; 324 participants). Subgroup analysis was performed according to whether or not random sequence generation had been reported. Three studies ${ }^{(15-17)}$ reported on random sequence generation comparing CMI plus CT with CT alone. The results showed that SIRS score decreased 1.05 points $(95 \% \mathrm{Cl}:-1.36$ to $-0.73, I^{2}=0$; 236 participants) in the treatment group. The other subgroup only mentioned random contained 1 study. ${ }^{(20)}$ The results are shown in Figure 3.

\section{APACHE II Score}

The APACHE II score was assessed by clinician in 4 studies, ${ }^{(20,22,27,33)}$ but 1 study $^{(33)}$ cannot be merged the data with other studies. Participants receiving

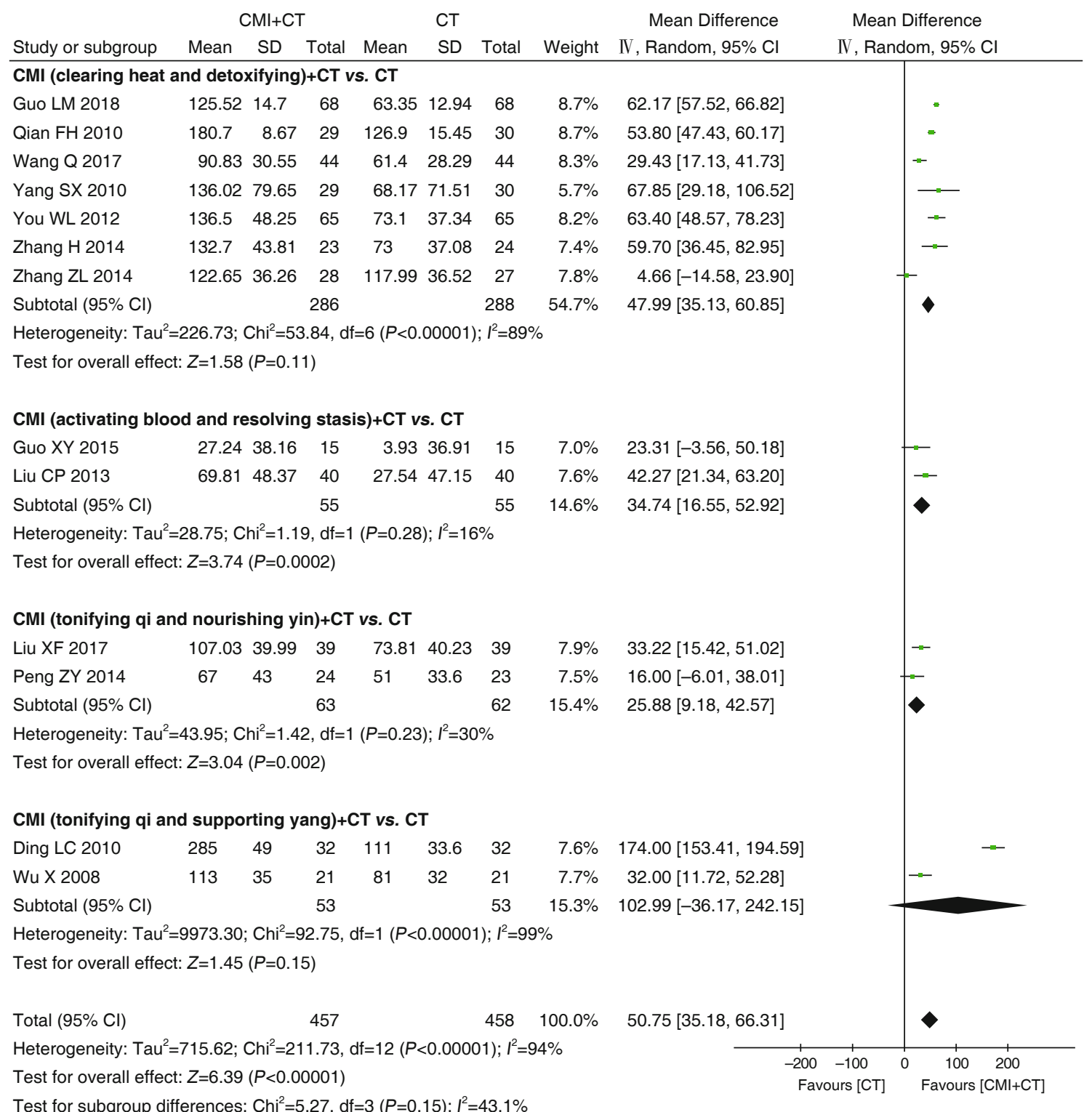

Figure 2. Comparison of CMI plus CT versus $\mathrm{CT}$ for ALI/ARDS: Change in $\mathrm{PaO}_{2} / \mathrm{FiO}_{2}$ 
CMI plus CT showed a significant decrease in the APACHE II score (MD: -1.74 points, $95 \% \mathrm{Cl}$ : -2.77 to $-0.71, l^{2}=0 ; 195$ participants), when compared with those receiving CT only. The results are shown in Figure 4.

\section{Duration of Mechanical Ventilation}

Duration of mechanical ventilation was evaluated in 7 studies. $^{(20-23,25,26,32)}$ Five studies ${ }^{(20,23,25,26,32)}$ used days as time unit, and results of the forest plot showed a high heterogeneity (MD: -2.94 days, $95 \% \mathrm{Cl}:-4.68$ to $-1.21, \mathrm{I}^{2}=89 \%$; 277 participants). Subgroup analysis was performed according to the different types of CMI. Participants receiving Shenfu Injection plus $\mathrm{CT}$ showed a significant reduction in the duration of mechanical ventilation (MD: -3.99 days, $95 \% \mathrm{Cl}:-5.03$ to $-2.95, I^{2}=31 \% ; 106$ participants in 2 studies), ${ }^{(25,26)}$ when compared with those receiving CT. The other 3 studies ${ }^{(20,23,32)}$ are each distributed in three subgroups, participants receiving Tanreqing Injection plus $\mathrm{CT}$, Xuebijing Injection plus CT or Dengzhanxixin Injection (灯盛细辛注射液) plus CT was superior to
CT alone. Two studies ${ }^{(21,22)}$ used hours as time unit, and there was no significant difference in the duration of ventilator between CMI plus CT and CT alone (MD: $-12.44 \mathrm{~h}, 95 \% \mathrm{Cl}:-40.48$ to $15.60, \mathrm{l}^{2}=95 \% ; 115$ participants). These results are shown in Appendixes 6 and 7.

\section{Length of Hospital Stay}

Length of hospital stay was evaluated by clinician in 6 studies, ${ }^{(20,23,25,26,29,32)} \mathrm{CMI}$ plus CT was superior to $\mathrm{CT}$, and the heterogeneity was high (MD: -4.22 days, $95 \% \mathrm{Cl}:-6.49$ to $-1.95, \mathrm{I}^{2}=92 \%$; 357 participants). Subgroup analysis was conducted based on the treatment cycle to deal with heterogeneity. Participants receiving treatment cycle more than 7 days in 5 studies, ${ }^{(20,23,25,26,32)}$ the result of subgroup showed CMI plus CT was superior to CT (MD: -3.23 days, $95 \% \mathrm{Cl}$ : -3.95 to $-2.51, I^{2}=0 ; 277$ participants). Participants receiving treatment cycle less than 7 days in 1 study, ${ }^{(29)}$ CMI plus CT was better than CT in decrease the length of hospital stay (MD: -7.68 days, $95 \% \mathrm{Cl}:-8.55$ to -6.81). The results are shown in Appendix 8.

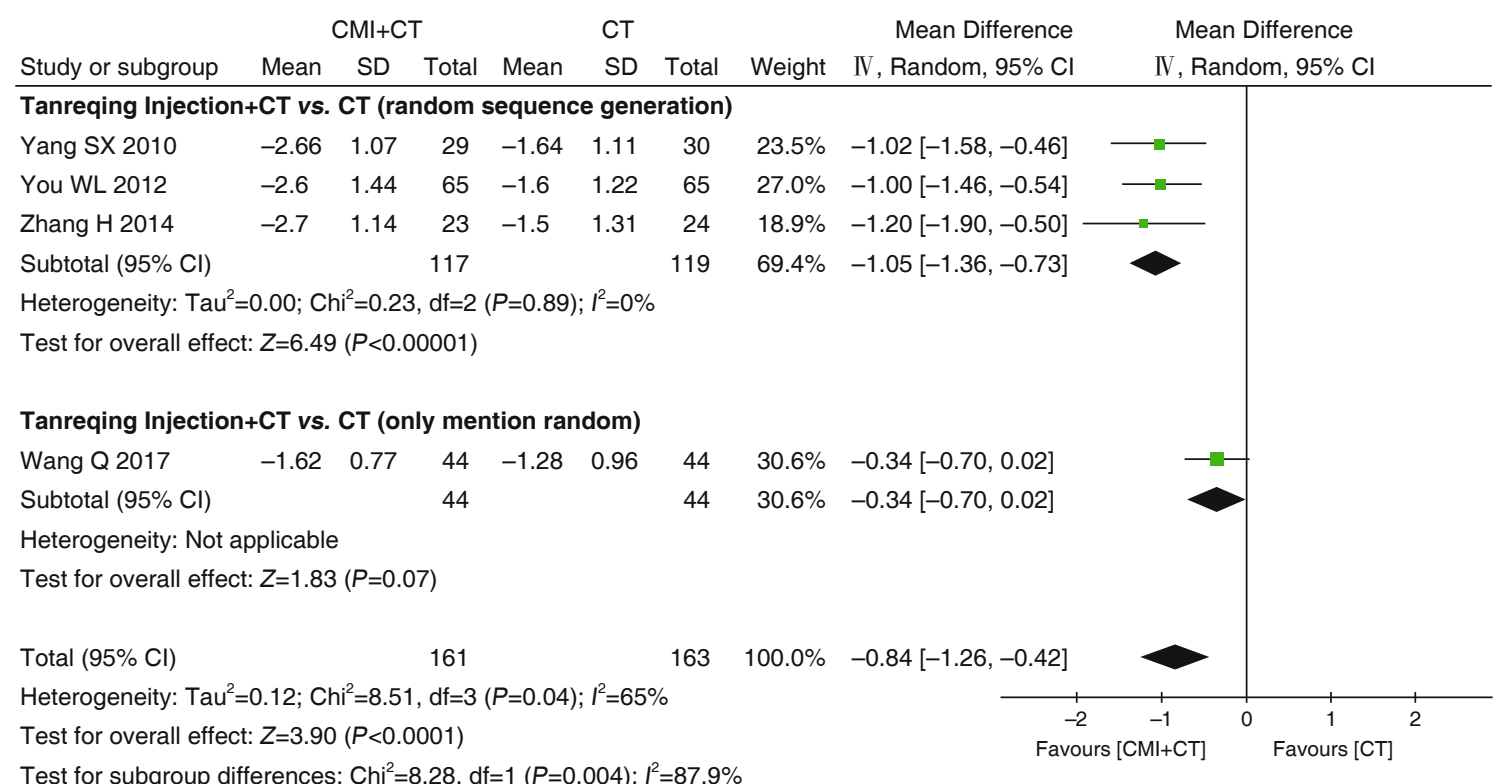

Figure 3. Comparison of CMI plus CT versus CT for ALI/ARDS: Change in SIRS Score

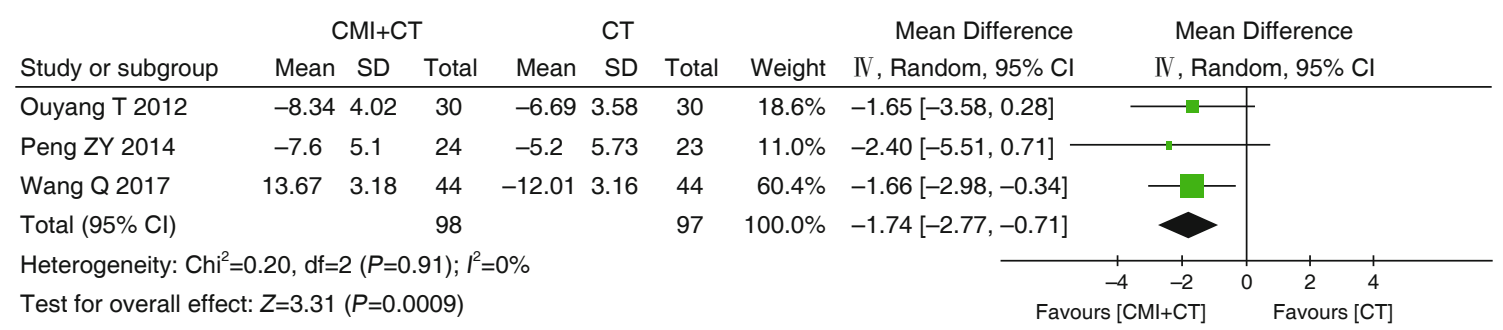

Figure 4. Comparison of CMI plus CT versus CT for ALI/ARDS: Change in APACHE II Score 


\section{Total Effective Rate}

Based on changes in symptoms, physical signs and related examinations after treatment, investigators judged the extent of the prognosis. It was then divided into 4 categories, including clinical controlled, markedly effective, effective and ineffective. ${ }^{(34,35)}$ To analyse the total effective outcome, patients that had reported any improvement were grouped together as effective.

Six studies ${ }^{(15,18,21,24,28,30)}$ had total effective rate as one of their outcomes. Participants receiving CMI plus CT showed a significant increase in total effective rate (RR: $1.35,95 \% \mathrm{Cl}: 1.17$ to $1.56, I^{2}=37 \% ; 290$ participants), when compared to those receiving CT alone. These results are shown in Appendix 9.

\section{Adverse Events}

Only 1 study ${ }^{(28)}$ mentioned adverse events, and no serious adverse events occurred. Nevertheless, reports of adverse events lacked sufficient detail.

\section{DISCUSSION}

This systematic review assessed 19 RCTs analysing a series of CMI for ALI/ARDS treatments. We also conducted meta-analyses to estimate the efficacy and safety of $\mathrm{CMI}$ according to outcome indicators such as $\mathrm{PaO}_{2}, \mathrm{PaO}_{2} / \mathrm{FiO}_{2}$, SIRS score, APACHE II score, duration of mechanical ventilation, length of hospital stay and total effective rate. It provides the latest evidence for ALI/ARDS alternative therapies. ALI/ARDS is an acute disease with a high mortality. At present, treatments based on Western medicine face many obstacles, leaving no ideal drug treatment. CMI intervention may provide a new therapeutic strategy for ALI/ARDS. Current evidence has demonstrated that $\mathrm{CMI}$ combined with $\mathrm{CT}$ is superior to $\mathrm{CT}$ alone as an adjunct therapy in improving clinical efficiency and reducing APACHE II score. Subgroup analysis showed that $\mathrm{CMI}$ for activating blood and resolving stasis plus CT plus CT was superior to $\mathrm{CT}$ in improving $\mathrm{PaO}_{2}$ and $\mathrm{PaO}_{2} / \mathrm{FiO}_{2}$. In terms of reducing the duration of mechanical ventilation, Shenfu Injection plus CT was better than $\mathrm{CT}$. When the treatment cycle exceeded 7 days, CMI plus CT was superior to CT in reducing the length of hospital stay. These results suggest that on the basis of $\mathrm{CT}$, combining $\mathrm{CMI}$ treatment could be a promising complementary therapy.

$\mathrm{CMI}$ is a new means of delivering treatment with its origins in China. It consists of a concoction of effective substances extracted from a single or compound $\mathrm{CM}$ prescription by modern scientific and technological methods, and guided by $\mathrm{CM}$ theory. ${ }^{(36,37)}$ Compared with other CM delivery methods such as decoction, pills and powder, CMI has the advantages of rapid absorption, high bioavailability, accurate positioning and high efficacy. CM researchers have made a great progress in the quality, safety, stability and controllability of $\mathrm{CMI}$ by improving preparation technology and quality control standards. CMI has been applied widely in clinic because it plays an important role in treating emergencies and severe disease.

ALI/ARDS is a common and devastating complication after acute illness or injury, it results in high morbidity and mortality. Dysregulated inflammation, coagulation and oxidative stress play central roles in ARDS pathophysiology. ${ }^{(38)}$ Both animal data and observational studies suggest CMI can modulate inflammation imbalance and abnormal coagulation process in ALI/ARDS. ${ }^{(39-41)}$ In China, Tanreqing Injection, Xuebijing Injection and Shenfu Injection have become common medicines in the clinical treatment of acute diseases. Research has shown that Xuebijing Injection may play a role in treating ALI by inhibiting inflammatory response and antioxidation, regulating abnormal coagulation and the fibrinolysis system. ${ }^{(42)}$ Tanreqing Injection has been shown to treat $A L I$ by antagonizing inflammation, up-regulating aquaporin and regulating the immune function. ${ }^{(43)} \mathrm{CMI}$ using the method of activating blood and resolving stasis showed better efficacy in reducing the APACHE II score in this review. CMI for activating blood and resolving stasis also showed good efficacy in improving $\mathrm{PaO}_{2}$ and $\mathrm{PaO}_{2} / \mathrm{FiO}_{2}$. Therefore, the above can explain the potential mechanism of $\mathrm{CMI}$ in treating ALI/ARDS. Complex CMI prescriptions have increasingly shown their advantages in treating ALI/ ARDS, and the mechanism is also being researched.

Current evidence suggests the paucity of effective therapeutic interventions in patients with ARDS, lung-protective ventilation and appropriate sepsis management seems to be the only strategies proven to improve outcomes in ARDS patients. ${ }^{(44)}$ Preventive strategies are a new field of research aiming to avoid the progression of ALI/ARDS. The key is to identify patients at risk of ALI/ARDS. This is consistent with the theoretical perspectives of preventive treatment of disease from $\mathrm{CM}$. It should be noted that we need to 
apply CMI correctly through syndrome differentiation. Early intervention of $\mathrm{CMI}$ seems to be a potential therapeutic strategy in the early treatment of ALI/ARDS.

In recent years, the application of CMI has been expanded, and adverse reactions due to it have gradually increased. ${ }^{(45,46)}$ Common adverse drug reactions attributable to $\mathrm{CMI}$ include allergic reaction, respiratory damage, digestive system damage and urinary system damage. Of these, allergic reaction is the most common. In some cases, it can even cause allergic shock. ${ }^{(4)}$ The main reason for CMI adverse reactions is that drug quality is not up to standards (i.e. standards are neither strict nor unified). For example, Tween-80 is commonly used as a cosolvent in CMI, which may be one of the main causes of clinical adverse reactions to various CMls. ${ }^{(48)}$ However, there are obvious discrepancies in quality control standards between countries. ${ }^{(49)}$ The second cause of adverse reactions is that the use of CMls is not standardized. Common problems include inaccurate diagnoses, improper compatibility with $\mathrm{CMs}$ and irregular medication combinations. ${ }^{(50)} \mathrm{CMI}$ is made by extracting the active ingredients of Chinese herbal medicine. It should only be used under the guidance of CM theory and should be based on syndrome differentiation.

How to reduce the incidence of adverse reactions of $\mathrm{CMI}$ is a crucible of exploration and development. Firstly, the Chinese government needs to formulate more stringent laws and regulations for the safe production and quality control of CMI. Secondly, the use of CMI should follow the CM theory of syndrome differentiation. Thirdly, the selection of CMI indications should be based on the results of evidence-based medicine. ${ }^{(51)}$

Only 1 study ${ }^{(28)}$ in this systematic review reported adverse events. This suggests that there remains insufficient evidence to support the safety of CMI. The safety risk of CMI in the treatment of ALI/ARDS is unclear. The reason may be that researchers still pay insufficient attention to the safety of CMI. Therefore, we strongly recommend that follow-up studies should report related adverse events. With the attention of the government and researchers, CMI will continue to improve. It is believed that $\mathrm{CMI}$ safety will also improve under the premise of reasonable use. ${ }^{(52)}$

The diagnostic criteria of included studies were reference to the AECC definition or Berlin definition. The
AECC definition was commonly used to examine the epidemiology and outcomes associated with ALI/ARDS in many countries. Nevertheless, it was suspected that this definition may not reflect the true prevalence, severity, and prognosis of ALI/ARDS because of several shortcomings. First, it failed to define timing of an acute onset. ${ }^{(53)}$ Second, it also failed to account for the effects of the positive end expiratory pressure (PEEP) which may change $\mathrm{PaO}_{2} / \mathrm{FiO}_{2}{ }^{\left({ }^{(54)}\right.}$ Moreover, patients with ARDS may have also elevated pulmonary artery wedge pressure. $^{(55,56)}$ In 2012, the definition of ARDS was revised, which is known as the Berlin definition. Berlin definition addresses some of the limitations of the AECC standard including absence of clear criteria for defining "acute onset," failure to account for different ventilator settings, and the poor reliability of the chest radiograph criterion. ${ }^{(53)}$ However, this new definition may still have limitations. Differences in risk factors, etiology, pathophysiology and prognosis between adults and children with ARDS were not considered. In addition, PEEP, pulmonary dead-space, extravascular lung water and biomarkers are important variables for clinicians to measure and understand in ARDS, but they are not included to predictive validity of severe ARDS definition. Most of the included studies adopt the AECC definition in this review, only 3 studies $^{(28,32,33)}$ adopt the Berlin definition. Because all studies were conducted in China, and the current guidelines for ALI/ARDS in China were derived from the AECC definition. Therefore, there may be a potential limitation in diagnosis of ALI/ARDS.

The reduction of selective bias for an $\mathrm{RCT}$ depends on appropriate randomization and allocation concealment. We noted that explicit random sequence generation was used in only $42 \%$ of the studies, none of which reported sufficient allocation concealment. This may have been due to the low methodological quality or poor quality of reports. Therefore, both may have exaggerated the efficacy. ${ }^{(57,58)}$ Inadequate blinding can also lead to high risk of performance bias, thus causing an overestimate effect. Although the blinding of participants and personnel is recommend in RCTs, it is difficult to accomplish in most cases because it requires a high-quality placebo (i.e. the same shape, smell, dosage form, etc.). In this systematic review, none of the studies used placebos or double blinding. This was due to the greater challenges presented by placebos and the blind implementation of injections. In addition, the included studies were single-centre RCTs with small sample sizes. This also limited our review. 
Therefore, multi-center, high-qualities RCTs of CMI for ALI/ARDS with large sample sizes are needed in the future.

Overall, evidence reveals benefits of $\mathrm{CMI}$ combined with CT for ALI/ARDS - it can reduce APACHE II score and improve clinical efficacy. However, the effects of $\mathrm{CMI}$ in improving $\mathrm{PaO}_{2}$ and $\mathrm{PaO}_{2} / \mathrm{FiO}_{2}$, reducing the duration of mechanical ventilation and length of hospital stay cannot be determined, due to heterogeneity. The safety risk of CMI for ALI/ARDS is still uncertain, because only 1 study mentioned relevant safety reports. The application of CMI in the treatment of ALI/ARDS is controversial, and the current evidence cannot give a positive conclusion. Future studies should focus on improving research design, especially blindness and placebo, and also reporting adverse events.

\section{Conflict of Interest}

The authors declare no conflicts of interest, and are responsible for the content and writing of the paper.

\section{Author Contributions}

Lin L initiated and supervised the study. Chen YB and Liu $Q$ conceived the project, analyzed the data and drafted the manuscript. Xie $\mathrm{H}$ and Yin SM searched the electronic databases and screened and extracted the studies. $\mathrm{Wu} \mathrm{L}$ and $\mathrm{Yu} \mathrm{XH}$ evaluated the risk of bias, and interpreted the evidence from a clinical perspective. Fan $L$ participated in the revision of the manuscript. All authors read and approved the final manuscript.

\section{Acknowledgments}

The authors thank Bo Li and evidence based clinical club for their help with methodological guidance.

Electronic Supplementary Material Supplementary material (Appendixes 1-9) is available in the online version of this article at http://dx.doi.org/10.1007/s11655-019-3078-7

\section{REFERENCES}

1. Bernard GR, Artigas A, Brigham KL, et al. The AmericanEuropean Consensus Conference on ARDS. Definitions, mechanisms, relevant outcomes, and clinical trial coordination. Am J Respir Crit Care Med 1994;149:818-824.

2. The ARDS Definition Task Force. Acute respiratory distress syndrome, the Berlin definition. JAMA 2012;307:2526-2533.

3. Rubenfeld GD, Caldwell E, Peabody E, et al. Incidence and outcomes of acute lung injury. N Engl J Med 2005;353:1685-1693.

4. Fan E, Brodie D, Slutsky AS. Acute respiratory distress syndrome: advances in diagnosis and treatment. JAMA 2018;319:698-710.

5. Johnson ER, Matthay MA. Acute lung injury: epidemiology, pathogenesis, and treatment. J Aerosol Med Pulm Drug Deliv 2010;23:243-252.

6. Matthay MA, Zimmerman GA, Esmon C, et al. Future research directions in acute lung injury: summary of a National Heart, Lung, and Blood Institute working group. Am J Respir Crit Care Med 2003;167:1027-1035.

7. Fan E, Del SL, Goligher EC, et al. An official American Thoracic Society/European Society of Intensive Care Medicine/Society of Critical Care Medicine clinical practice guideline: mechanical ventilation in adult patients with acute respiratory distress syndrome. Am J Respir Crit Care Med 2017;195:1253-1263.

8. Matthay MA, Zimmerman GA. Acute lung injury and the acute respiratory distress syndrome: four decades of inquiry into pathogenesis and rational management. Am J Respir Cell Mol Biol 2005;33:319-327.

9. Levy BD, Clish CB, Schmidt B, et al. Lipid mediator class switching during acute inflammation: signals in resolution. Nat Immunol 2001;2:612-619.

10. Calfee CS, Matthay MA. Nonventilatory treatments for acute lung injury and ARDS. Chest 2007;131:913-920.

11. Li YK, Zhang JY. The improvement and enhancement of traditional Chinese medicine injections. China J Chin Mater Med (Chin) 2011;36:1905-1909.

12. Xue LX, Chen JR, Tao YJ, et al. Effects of Xuebijing on nitric oxide and VEGF-A in exhaled breath condensate of patients with ALI/ ARDS. Chin J Integr Tradit West Med (Chin) 2013;33:766-799.

13. Yang SX, Zhao YL, Zeng JY. Recent application of Tanreqing Injection in acute lung injury. Intern Med (Chin) 2009;4:918-921.

14. Higgins JPT, Green S. Cochrane handbook for systematic reviews of interventions version 5.3.3 (updated 2016). The Cochrane Collaboration.

15. Zhang $\mathrm{H}$. The clinical study of Tanreqing Injection in acute lung injury. Med People (Chin) 2014;27:70.

16. You WL. The clinical randomized-controlled study of Tanreqing Injection in acute lung injury. J Clin Pulm Med (Chin) 2012;17:1987-1988.

17. Yang SX, Zhao YL, Zeng JY, et al. Clinical observation of Tanreqing Injection on acute lung injury patients. Guangxi Med J (Chin) 2010;32:144-147.

18. Zhang HX, Bao N, He YS. Application of Tanreqing Injection in acute lung injury. J Emerg Tradit Chin Med (Chin) 2013;22:639-640.

19. Guo LM, Guo S, Ma HH. Effect of Tanreqing Injection on levels of inflammatory mediators in patients with acute lung injury. Chin J Integr Tradit West Med Intensive Crit Care (Chin) 2018;25:242-245.

20. Wang Q. Effects of bronchoalveolar lavage with dexamethasone combined with Tanreqing Injection on cytokines in serum and broncho-alveolar lavage fluid in patients with acute lung injury. Mod J Integr Tradit Chin West Med (Chin) 2017;26:2093-2096.

21. Zhang ZL, Lin H, Wang GH, et al. Study on the protective effect of heat-clearing and phlegm-resolving method on acute lung injury patients with syndrome of phlegm-heat accumulation in Lung. Lishizhen Med Mater Med Res (Chin) 2014;25:2444-2446.

22. Ouyang T, Ouyang YP, Wei QJ. The affects on the Xuebijing Injection against respiratory function and early inflammatory response in patients with ALI. Clin J Chin Med (Chin) 2012;4:6-8.

23. Bai SX. Clinical observation of Xuebijing in patients with severe 
pneumonia complicated with ARDS. Shandong Med J (Chin) 2014;54:61-62.

24. Wang XD, Li M, Zhang C, et al. Effects of Xuebijing in patients with acute respiratory distress syndrome. J Xuzhou Med Univ (Chin) 2007;27:732-735.

25. Wu X, Li WF, Liu XF. Effects of Shenfu Injection on extravascular lung water and oxygenation in patients with ARDS. J Emerg Tradit Chin Med (Chin) 2008;17:1702-1704.

26. Wang LC, Liu XF, He C, et al. Study of Shenfu Injection on blood flow volume of lungs and breathing mechanics in ARDS. J Emerg Tradit Chin Med (Chin) 2010;19:774-775,792.

27. Peng $Z Y$, Peng HQ, Lin HW, et al. Effect on acute lung injury in patients with sepsis by supplementing qi nourishing yin and promoting blood flow. J Liaoning Univ Tradit Chin Med (Chin) 2014;16:168-170.

28. Liu XF. Clinical effects and ICAM-1, ET-1 and NO levels of Shengmai Injection in treatment of acute lung injury. Mod J Integr Tradit Chin West Med (Chin) 2017;26:2784-2786.

29. Shi $\mathrm{CH}$, Zhang JB, Xu GB, et al. Effect and safety of Danshen Injection in the treatment of patients with acute lung injury. Chin J Clin Pharmacol (Chin) 2013;29:501-502.

30. Qian FH, Qian YM, Zhu L, et al. The clinical effect of Reduning Injection on acute lung injury. Chin J Prim Med Pharm (Chin) 2010;17:1318-1319.

31. Liu CP, Hu P, Li CH, et al. Therapeutic value of aescine in traumatic acute lung injury. J Trauma Surg (Chin) 2013;15:497-499.

32. Guo XY, Chen RY, Zhang Y. Effect of Erigeron Breviscapus Injection on inflammatory factors and clotting mechanism in ARDS patients. J Emerg Tradit Chin Med (Chin) 2015;24:854-856.

33. Meng L, Chen HL, Lin HW. Effects of Rhodiola Wallichiana Injection on clinical efficacy and the concentration of VEGF-A of EBC in patients with ALI/ARDS. J Clin Emerg (Chin) 2018;19:97-101.

34. Zheng XY, ed. Guiding principle of clinical research on new drugs of traditional Chinse medicine. Beijing: Medic-Pharmaceutical Sciences and Technology Publishing House;2002:54-60.

35. The State Administration of Traditional Chinese Medicine. Criteria for the diagnosis and therapeutic effect of Chinese medicine on dyspnea. J Liaoning Univ Tradit Chin Med (Chin) 2015;17:67.

36. The Ministry of health, the State Food and Drug Administration and the State Administration of traditional Chinese Medicine. Notice on further strengthening the administration and administration of traditional Chinese medicine injections. People's Republic of China State Health and Family Planning Commission Bulletin;2009:64-65.

37. Han W, He E, Cao J. Application of traditional Chinese medicine injections using traditional Chinese medicine theory. Chin J Chin Mater Med (Chin) 2012;37:2498-2500.

38. Matthay MA, Ware LB, Zimmerman GA. The acute respiratory distress syndrome. J Clin Invest 2012;122:2731-2740.

39. Chen JW, Lin HX, Li SW. A clinical study on ulinastatin combined with Salvas Miltiorrhiza in adjuvant treatment of acute lung injury. Int Med Health Guid News (Chin) 2013;19:322-324.

40. Luo $P$, Zhou ZX. Protective effects of Xuebijing on the acute lung injury in rats. Chin J Appl Physiol (Chin) 2017;33:132-135.

41. Tang LP, Xiao W, Li YF, et al. Anti-inflammatory effects of Reduning Injection on lipopolysaccharide-induced acute lung injury of rats. Chin J Integr Med 2014;20:591-599.

42. Wang $P$, Zhao HD. Study of Xuebijing on the treatment mechanism of acute lung injury. Chin J Crit Care Med (Chin) 2010;30:79-81.

43. Zhuang XF, Yu SL, Fan ML, et al. Advances on mechanism research of traditional Chinese prescription for the treatment of acute lung injury. Chin J Exp Tradit Med Form (Chin) 2012;18:326-331.

44. de Haro C, Martin-Loeches I, Torrents E, et al. Acute respiratory distress syndrome: prevention and early recognition. Ann Intensive Care 2013;3:11.

45. Bian ZX, Tian HY, Gao L, et al. Improving reporting of adverse events and adverse drug reactions following injections of Chinese materia medica. J Evid Based Med (Chin) 2010;3:5-10.

46. State Food and Drug Administration. Notice on the revaluation of safety of traditional Chinese medicine injections. Adv Drug React J (Chin) 2009;11:353-360.

47. Kang RX, You RL, Wang L, et al. In vitro experiment of allergic reactions induced by traditional Chinese medicine injections. Chin J Chin Mater Med (Chin) 2015;40:2503-2507.

48. Li Y, Zhang J. Improvement and enhancement of traditional Chinese medicine injections. Chin J Chin Mater Med (Chin) 2011;36:1905-909.

49. Zhang M. Advance of polysorbate 80 for injection accessories. Chin J Chin Mater Med (Chin) 2011;36:1910-191.

50. Xiong XJ, Wang J, He QY. Application status and safety counter measures of traditional Chinese medicine injections. J Chin Integr Med (Chin) 2010;8:307-311.

51. Ji K, Chen J, Li M, et al. Comments on serious anaphylaxis caused by nine Chinese herbal injections used to treat common colds and upper respiratory tract infections. Regul Toxicol Pharmacol (Chin) 2009;55:134-138.

52. Chen F, Liu Z, Safety evaluation of traditional Chinese medicine injections and study of related key technology. Chin J Chin Mater Med (Chin) 2009;34:1052-1054.

53. Sine CR, Belenkiy SM, Buel AR, et al. Acute respiratory distress syndrome in burn patients: a comparison of the Berlin and AmericanEuropean definitions. J Burn Care Res 2016;37:e461-e469.

54. Villar J, Perez-Mendez L, Lopez J, et al. An early PEEP/FiO trial identifies different degrees of lung injury in patients with acute respiratory distress syndrome. Am J Respir Crit Care Med 2007;176:795-804.

55. Ferguson ND, Meade MO, Hallett DC, et al. High values of the pulmonary artery wedge pressure in patients with acute lung injury and acute respiratory distress syndrome. Intensive Care Med 2002;28:1073-1077.

56. Wheeler AP, Bernard GR, Thompson BT, et al. Pulmonaryartery versus central venous catheter to guide treatment of acute lung injury. N Engl J Med 2006;354:2213-2224.

57. Hopewel S, Clarke M, Moher D, et al. CONSORT for reporting randomized controlled trials in journal and conference abstracts: explanation and elaboration. PLoS Med 2008;5:48-56.

58. Pildal J, Hróbjartsson A, Jørgense KJ, et al. Impact of allocation concealment on conclusions drawn from meta-analyses of randomized trials. Int J Epidemiol 2007;36:847-857.

(Accepted March 29, 2019; First Online November 28, 2019) Edited by YU Ming-zhu 